\title{
Biomonitoring with Wireless Communications
}

\section{Thomas F. Budinger M.D., Ph.D.}

Affiliations: 1) Department of Functional Imaging, Lawrence Berkeley National Laboratory;

Berkeley, CA. 94720; USA

2) Departments of Bioengineering and Electrical Engineering and Computer Science, University of California, Berkeley; CA. 94720; USA

3) Department of Radiology, University of California, San Francisco, CA. 94143; USA

Email: tfbudinger@lbl.gov

Key Words: Physiologic Monitoring, Fall detection, Tracking monitors, Local Area Network (LAN), Biosensors

Running Head: $\quad$ Biomonitoring

Correspondence should be sent to: Dr. Thomas Budinger

LBNL

1 Cyclotron Road. Mailstop 55-121

Berkeley, CA. 94720 USA

Phone: (510) 486-4074

Fax: (510) 486-4768

Email: tfbudinger@lbl.gov 


\section{Biomonitoring with Wireless Communications \\ Thomas F. Budinger M.D., Ph.D.}

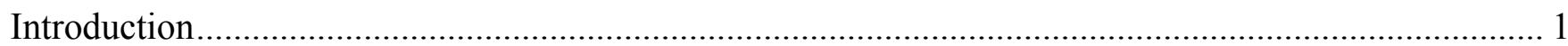

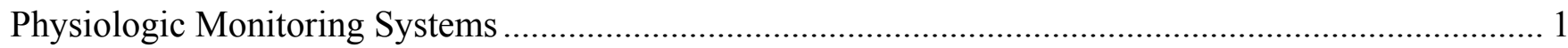

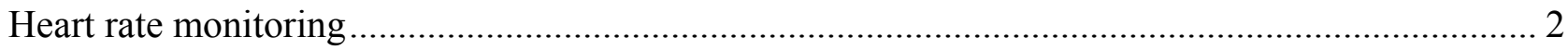

Blood Oxygenation / Pulse Oximetry ………………............................................................ 4

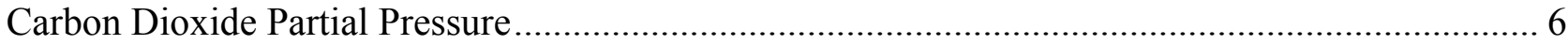

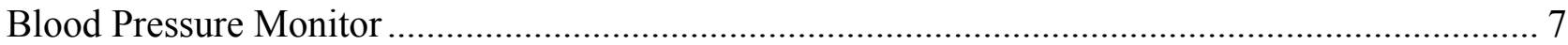

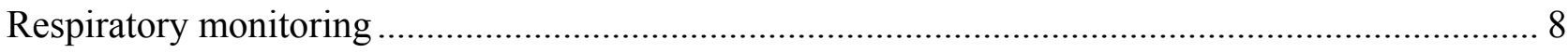

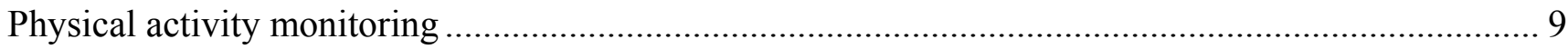

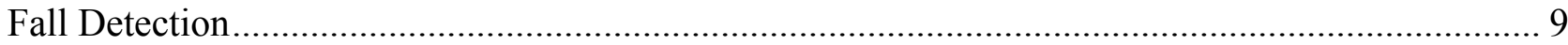

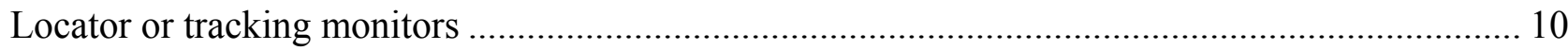

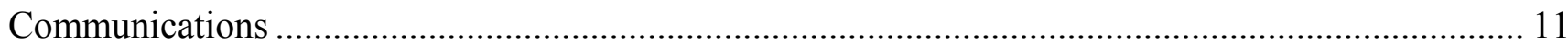

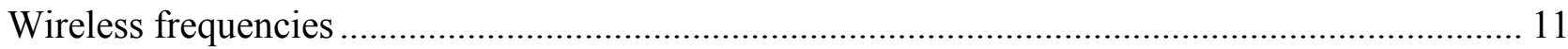

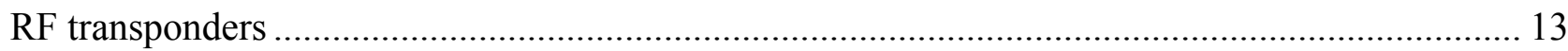

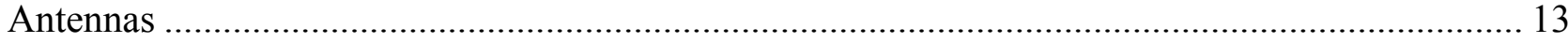

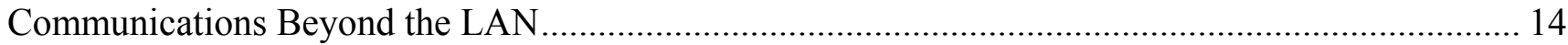

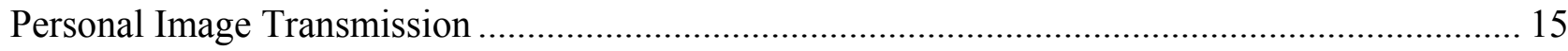

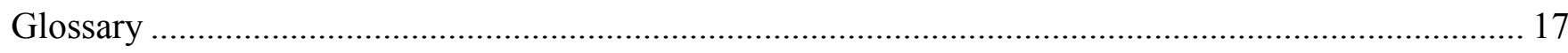

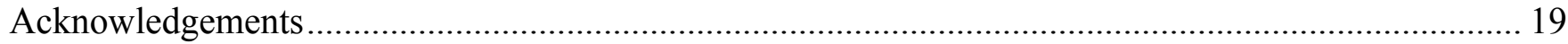

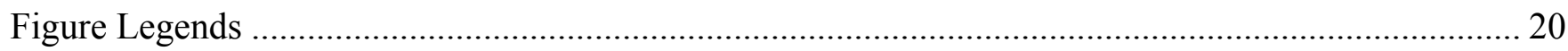

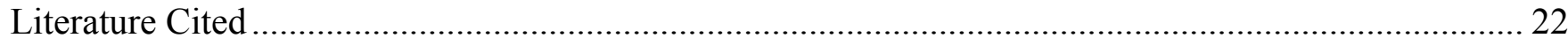

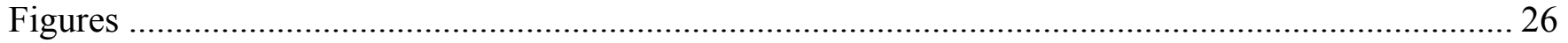




\section{Biomonitoring with Wireless Communications}

\section{Thomas F. Budinger M.D., Ph.D.}

\section{Introduction}

This review is divided into three sections: technologies for monitoring physiological parameters; biosensors for chemical assays and wireless communications technologies including image transmissions. Applications range from monitoring high risk patients for heart, respiratory activity and falls to sensing levels of physical activity in military, rescue, and sports personnel. The range of measurements include, heart rate, pulse wave form, respiratory rate, blood oxygen, tissue $\mathrm{pCO}_{2}$, exhaled carbon dioxide and physical activity. Other feasible measurements will employ miniature chemical laboratories on silicon or plastic chips. The measurements can be extended to clinical chemical assays ranging from common blood assays to protein or specialized protein measurements (e.g. troponin, creatine, and cytokines such as TNF and IL6). Though the feasibility of using wireless technology to communicate vital signs has been demonstrated 32 years ago (1) it has been only recently that practical and portable devices and communications net works have become generally available for inexpensive deployment of comfortable and affordable devices and systems.

\section{Physiologic Monitoring Systems}

The variety of sensors which meet the criteria of non-invasiveness, comfort, practical, and medically useful are summarized in Figure 1. Not all of these monitoring devices or ideas have been reduced to practical devices but those which are not available are either currently in research or have plausibility from engineering perspectives. 
Budinger TF. Biomonitoring with wireless communications. Annu Rev Biomed Eng 2003; 5:383-412. Though the focus of this review is on biomonitors, biosensors and wireless communications, one should not neglect the substantial background technology for bedside monitoring of chronic and acute status by well known devices. Common measurements include ECG, temperature, blood pressure, oxygen saturation, blood flow and respiratory rate. Some of these systems have wireless modules, but the development of LAN in hospitals has not matured. The performance of these systems has been under comparative review as found in the periodical Health Devices (2).

\section{Heart rate monitoring}

Heart rate or pulse rate monitoring can be accomplished by a number of methods some of which are appropriate for non-electrode based wireless communications. These methods include:

1) wireless heart rate chest strap,

2) electrical wave form (ECG),

3) pulse oximeter,

4) ultrasound,

5) pulse pressure detection by strain gauge impedance or piezoelectric volume change,

6) electromagnetic flow and

The most frequently used method by athletes is the chest strap monitor which transmits \#?? heart rate signals collected by 2 electrodes across the chest which pick up the potential differences in rate with ?? (e.g., Nordic Track (www.nordictrack.com )). The signal is detected within 2-3 meters by a wrist watch or electronics in exercise equipment (e.g., treadmills, bicycles ).

Reviews of most of these methods are found in Medical Instrumentation: application and design (3) and the Handbook of Bioengineering (4). In addition to pulse rate, these methods provide important Biomonitoring page 2 
Budinger TF. Biomonitoring with wireless communications. Annu Rev Biomed Eng 2003; 5:383-412. information in their respective waveforms. The information contained in the electrical signal is very different from that in the pulse pressure time variation or the blood velocity signals. All methods give heart rate but the information obtainable from the pressure pulse and the ECG are sufficiently different that innovations which allow convenient acquisition of these data might prove to be very valuable even in monitoring non-high risk subjects (e.g. those recovering from illnesses or patients undergoing recuperative chemical therapies). Figure 2 diagrams these differences as will as how a combination of two methods can give pulse transit time information which will be discussed later.

One of the earliest examples of wireless medical telemetry is fetal heart rate and intra uterine pressure monitoring by probes inserted into the uterus with a two-channel miniature radio transmitter. Signals detected by a receiver at distances of about 10 meters allow wireless monitoring (1). This type of monitoring are in current practice with wired connections to modern bedside equipment. Indeed hardwired systems in hospital environments are the practice and there is not a clear need for wireless connections in intensive or critical care situations, within the local hospital environment. Of course beyond the critical care nurses stations, there is a major problem of communications to the cardiologist or other caregivers of data such as ECG, intra uterine pressure curves, oxygen saturation, and other parameters which cannot be reliably communicated by voice to physicians. It is the flexible and accurate communication remote from the bedside, which requires a robust communications net work. Within the hospital environment where wiring beds in a ward is awkward, wireless systems to a LAN have been shown to be effective (5). Outside the hospital a number of systems have shown successful operations recently. Examples are ECG consultation with cardiologists using pocket wireless computers (6) and a home based systems for cardio-respiratory monitoring of elderly or ill patients (7). Beyond the home and hospital environments, that is in space or environments remote from cosmopolitan networks there have been remarkable successes as evidenced by the Mt Everest experiment (8), the commercial airline ECG transmission experiment (9) and the recent simulation of a Mars medical data communication link from the Arctic remote environment (10). 


\section{Blood Oxygenation / Pulse Oximetry}

Tissue oxygen saturation as reflected in circulating blood oxygen levels is a variable of major importance in acute patient monitoring in the hospital and at the scene of accidents. The simplest method is by pulse oximetry whereby the differential absorbency of light through the capillary bed of the ear lobe or finger tip can give a reasonably reliable parameter which allows continuous monitoring. Most systems are for bedside monitoring (e.g. Welch Allyn (www.monitoring.welchallyn.com)) and there has been little demand for miniature portable systems

The measurement is performed at two specific wavelengths: a wavelength of about $660 \mathrm{~nm}$ where there is a large difference in light absorbance between $\mathrm{Hb}$ and $\mathrm{HbO}_{2}$, and a second wavelength typically chosen between 805 and $960 \mathrm{~nm}$ in the near infrared region. Around $805 \mathrm{~nm}$ the absorbance of light is about equal for $\mathrm{Hb}$ and $\mathrm{HbO}_{2}$. A measurement of the percent oxygen saturation of blood in vitro is made by comparing the log of the transmitted to emitted light power or the optical densities at the two wavelengths. A measurement in vivo must take into account the light absorption by the venous blood and the bloodless tissues. This is accomplished by comparing the AC optical densities to the DC densities over the two wavelengths. These densities are determined from the time vs transmitted light signals obtained by rapidly pulsing light at the two successive wavelengths during the cardiac cycles. Thus the name pulse oximeter.

The pulse oximetry signal is caused by changes in arterial blood volume associated with each heart beat. The magnitude of this signal depends on the amount of blood pulsing into the peripheral vascular bed with each cardiac cycle, the optical absorption of the blood, skin, and tissue, and the wavelength used to illuminate the blood. $\mathrm{S}_{\mathrm{a}} \mathrm{O}_{2}$ is derived by analyzing the magnitude of the $660 \mathrm{~nm}$ and the longer wavelength transmission measured by the photodetector. Electronic circuits separate their signals into pulsatile (AC) and non-pulsatile (DC) signal components. An algorithm inside the pulse oximeter performs a mathematical normalization by which the AC signal at each wavelength is divided by the 
Budinger TF. Biomonitoring with wireless communications. Annu Rev Biomed Eng 2003; 5:383-412. corresponding DC component. The DC component is related to absorption by the bloodless tissue, residual arterial blood when the heart is in diastole, venous blood, and skin pigmentation. Since it is assumed that the AC portion of the photoplethysmogram results only from the arterial blood component, this scaling process provides a normalized red / infrared ratio, R. which is highly dependent on the color of the arterial blood (i.e., $\mathrm{S}_{\mathrm{a}} \mathrm{O}_{2}$ ) but is largely independent of the volume of arterial blood entering the tissue during systole, skin pigmentation, skin thickness, and vascular structure. Therefore, the instrument does not need to be recalibrated for measurements on different patients.

A wrist worn pulse oximeter engineered by Minolta is shown in Figure 3. This system measures both pulse rate and blood oxygen saturation with an accuracy of $\sim 2 \%$. The readout is a back-lighted display from the finger tip sensor. This is probably the smallest portable commercial system with a battery life of $48 \mathrm{hr}$. (2 AAA) and mass of $42 \mathrm{~g}$. Wireless systems have not yet been developed. The device in Figure 5 does provide a platform for collecting data which can be sent conveniently through wireless LAN, either directly to repeaters in the hospital or home environment or indirectly via the persons on board LAN.

Highly precise arterial oxygen saturation ( $\mathrm{SaO} 2)$ measurements are commonly obtained using transmittance pulse oximeters in clinical situations (11). The application site of the transmission pulse oximeters is limited mainly to the peripheral tissue, such as finger tip, ear lobe or toe from which the transmitted light can be detected. Alternately, a reflectance oximeter could measure $\mathrm{SaO} 2$ from various parts of the body, especially from other body regions such as the forehead, cheek, wrist etc. With the reflectance oximeter, the wavelength combination, $730 / 880 \mathrm{~nm}$, was determined to obtain a linear relationship between the reflectance ratio and the broader $\mathrm{SaO} 2$ range from $100 \%$ to $30 \%$ in comparison to the $665 / 910 \mathrm{~nm}$ conventional wavelength (12). The reflection pulse oximeter sensor can be applied to various locations of the body in the hospital and home health care to aid in the early diagnosis of cardiopulmonary as well as peripheral circulatory disorders. 


\section{Carbon Dioxide Partial Pressure}

Transcutaneous partial pressure of $\mathrm{CO}_{2}$ can be measured by a portable system (13) similar to that diagrammed in Figure 4. The $\mathrm{CO}_{2}$ sensor is a glass $\mathrm{pH}$ electrode with a concentric $\mathrm{Ag} / \mathrm{AgCl}$ reference electrode that is used as a heating element. The electrolyte, a bicarbonate buffer, is placed on the electrode surface. $\mathrm{A} \mathrm{CO}_{2}$-permeable Teflon membrane separates the sensor from its environment.

The transcutaneous $\mathrm{pCO}_{2}$ sensor operates according to the Stow-Severinghaus principle: that is, a $\mathrm{pH}$ electrode senses a change in the $\mathrm{CO}_{2}$ concentration. This system is calibrated with a known $\mathrm{CO}_{2}$ concentration solution.

Heating the skin beneath the sensor causes increase in measured

1) $\mathrm{pCO}_{2}$, because the solubility of $\mathrm{CO}_{2}$ in tissue decreases with an increase in temperature;

2) local tissue metabolism, because cell metabolism is directly correlated with temperature;

3) the rate of $\mathrm{CO}_{2}$ through the stratum corneum, which increases with temperature.

As a consequence of these three effects, which all work in the same direction to increase transcutaneous $\mathrm{pCO}_{2}$ values, heating the skin yields $\mathrm{pCO}_{2}$ values larger than the corresponding arterial $\mathrm{pCO}_{2}$. Nevertheless the correlation between transcutaneous $\mathrm{pCO}_{2}$ and arterial $\mathrm{pCO}_{2}$ is usually satisfactory. Because the slope of the $\mathrm{CO}_{2}$ electrode calibration line is essentially that of the Nernst equation, a twopoint calibration is not needed (14). Transcutaneous $\mathrm{pCO}_{2}$ sensors have responses in the order of minutes which depend on the induced skin temperature with a time of 3.5 minutes for the maximum temperature one would reasonably achieve of $44^{\circ} \mathrm{C}(15)$. 


\section{Blood Pressure Monitor}

Currently the most portable, user friendly blood pressure measurement device is an applied monitor marketed by OMRON Inc (www.omron.com) and shown in Figure 5. These units have a 14 memory storage feature which makes recording measurements easy, They are powered on 2 AAA batteries and can measure blood pressure and pulse. The wrist cuff automatically inflates and deflates and these devices have been proven clinically accurate.

\section{$\underline{\text { Pulse Transit Time }}$}

Pulse transit time (PTT) refers to the time it takes a pulse wave to travel between two arterial sites. The speed at which this arterial pressure wave travels is inversely proportional to vascular compliance and since there is a direct relationship between vascular tone stiffening and acute rises in blood pressure, PTT decreases are proportional to blood pressure increases. Conversely, when blood pressure falls, vascular tone decreases and PTT increases.

Originally PTT was measured by recording the time interval between the passage of the arterial pulse wave at two consecutive sites. More recently, for ease of measurement, the electrocardiographic R or Q wave has been used as the starting point as it corresponds approximately to the opening of the aortic valve (Figure 2). The interval between the $\mathrm{R}$ wave and the arrival of the pulse wave at a peripheral site, such as the finger using photo-plethysmography, is the PTT (Figure 2). Using ECG leads and finger photo-plethysmography reproducible PTT measurements can be made very simply.

There is currently a need to simplify tests used in the investigation of patients with suspected sleep disturbed breathing without necessarily compromising the accuracy of their findings. The capability of PTT to identify and semi-quantitatively measure respiratory effort has been established (16). 


\section{Respiratory monitoring}

One of the first successful deployments of a wireless breathing monitor is the radio transmitter / receiver system for monitoring breathing and other sounds of infants in the home. These systems operate at 900 $\mathrm{MHz}$ and give reliable communications within ranges of 300 meters at affordable prices of $\sim \$ 60$ (e.g. www.babyuniverse.com).

The major motivation for development of monitoring systems for breathing has been preventative medicine for sudden infant death syndrome (SIDS) which appears to be related to apnea. Respiration patterns are measured by direct wire techniques.

The combination of respiratory monitor and wireless technologies is embodied in a commercially available cardiorespiratory monitor working with field plethysmography, wireless signal transmission and an alarm system (SpiroGuard C). In order to determine the recognition rates for central, mixed and obstructive apneas, a prospective clinical trial was performed comparing frequency and kind of signals from the monitor with those simultaneously registered by polysomnographic studies. Approximately half of the alarms were false alarms. These could be reduced by setting the apnea detection time to $>15$ seconds, by tighter fastening of the respiration belt (improving the signal transmission), and by turning off the instrument when the child is awake and physically active. The wireless system renders the SpiroGuard $\mathrm{C}$ an attractive alternative for home monitoring (17).

A device for infant monitoring of multiple parameters has been developed for the Collaborative Home Infant Monitoring Evaluation (CHIME). A new physiologic monitor for use in the home has been developed and used for the Collaborative Home Infant Monitor Evaluation (CHIME). This monitor measures infant breathing by respiratory inductance plethysmography and transthoracic impedance; infant electrocardiogram, heart rate and R-R interval; hemoglobin $\mathrm{O} 2$ saturation of arterial blood at the periphery and sleep position. The monitor was considered to be superior to conventional monitors and, therefore, suitable for the successful conduct of the CHIME study (18). 


\section{Physical activity monitoring}

Sleep research objectives have motivated development of accelerometer based systems for measuring an individuals movements. These systems developed approximately 15 years ago are based on piezoelectric accelerometers with reliable performance even with only one dimensional capability. The devices are known as "actigraphs" and can be commercially acquired from a number of companies (e.g. Ambulatory Monitoring, Inc. New York iLifesystems, Oregon ). These devices are currently used for monitoring activity of patients and are being promoted as an accessory for the general population interested in having a metric of their exercise activity. The devices can be worn on the wrist or elsewhere such as the belt. A drawing to two types of these devices is shown in Figure 6. Though the implementation of wireless communication of the wrist mounted device is only on the drawing board at present the wireless communication of the larger belt-worn system information to a local LAN is part of the current product. The watch read-out is wireless through LED communication.

\section{Fall Detection}

Devices which allow patients at risk for cardiac events which require immediate response have been developed with wireless communication capabilities to a home based LAN with "burglar alarm" type communication to a commercial server. These devices serve a patient in distress but also have a major role in fall detection as falls are the leading cause of death by injury for people over 65 . Overall $33 \%$ of people over 65 will have a fall according to the National Safety Council and in a life time of individuals over 65 one third will eventually be disabled or killed by a fall. A remarkable further statistic is that at least 300,000 people are found dead or helpless in their own home s in the U. S. every year and approximately $10 \%$ of people who fall at home are on the floor for more than one hour (19). To meet this problem commercial activities have developed panic button or alert systems which depend on the home LAN for secure communications to a response center just as one would communicate by wireless network in the home a burglar entry or fire. One of these systems known as HealthSensor 100, 
Budinger TF. Biomonitoring with wireless communications. Annu Rev Biomed Eng 2003; 5:383-412. (Framingham, Mass.) uses a voice communicator links the patient or person in need to the response center. One such system is called Lifeline located in Framingham, Massachusetts. Two-way speakerphone capability allows communication between the patient and personnel at the call center. Panic buttons on a necklace or wrist worn device send an alarm which can be reset or cancelled but if not will activate a telephone communications between patient and communications central.

The Australia Commonwealth Scientific and Industrial Research Organization (CSIRO) has fielded a project called Hospital Without Walls, which aims to provide continuous monitoring of patients in certain diagnostic categories (20). The key technology is a miniature, wearable, low-power radio that can transmit vital sign and activity information to a home computer, from which data may be sent by telephone line and the internet to appropriate medical professionals. Accelerometers and radio transmitters worn on the patient use LAN to relay activity and characterize falls. Simultaneous measurement of heart rate can provide information about abnormalities of cardiovascular physiology at the time of a fall.

Web sites relevant to fall alerts and panic button communications are:

1) http://www.americanmedicalalarms.com

2) http://www.ilifesystems.com;

3) http://www.lifealert.com

4) $\quad$ http://www.seniorsafety.com.

\section{Locator or tracking monitors}

In the past 5 years a number of commercial devices have become available for the tracking of patients (e.g. Alzheimer's patients) children and pets. The oldest system is WorldTrack

(http://www.eworldtrack.com) which is designed to give the family, custodian and emergency/medical 
Budinger TF. Biomonitoring with wireless communications. Annu Rev Biomed Eng 2003; 5:383-412.

personnel a wireless system for locating individuals who have an on board global position system (GPS) receiver and a communication system to their own network. Other systems are about to be offered to the public by Siemens AG and Applied Digital Solutions (http:/www.digitalangel.net). Commercial systems require communications through a burglar alarm and fire alarm on the home central office such as is used for or in the case of the proposed Digital Angel system. Tracking can be done by the concerned family member via the internet. Of course there is a monthly fee for maintaining this service but the equipment needed other than the access to the internet requires less than $\$ 700$ investment for the on-board communication device. An alternative approach which could be deployed without reliance on commercial vendors is shown in Figure 7.

A wrist watch with a reasonably long range beacon has been offered by Breitling (Figure 8). On flat terrain or calm seas the transmitter's signal on the $121.5 \mathrm{MHz}$ aircraft emergency frequency has a range of about 160 kilometers (100 miles) assuming the search craft is flying at 6,000 m (about 20,000 feet).

Use of home or personal monitoring systems including fall alerts poses some safety problems. The implications of any failure of a technology on which patients or care-givers rely, must be addressed in order to provide a safe and reliable care service. This topic has been reviewed relative to procedures for risk assessment (21).

\section{Communications}

\section{Wireless frequencies}

The range of frequencies used for electromagnetic wireless communications are from $121 \mathrm{MHz}$ used in the Breitling Wrist Watch emergency beacon transmitter (Figure 8) to $2.5 \mathrm{GHz}$ commonly used in short rang local area networks (LAN). Common frequencies for HAM radio are $141 \mathrm{MHz}$ (2 meter wavelength) and $400 \mathrm{MHz}$ (ca.70 cm wavelength) and cellular phone frequencies are in the $900 \mathrm{MHz}$ (ca. $30 \mathrm{~cm}$ wavelength) range. 
Budinger TF. Biomonitoring with wireless communications. Annu Rev Biomed Eng 2003; 5:383-412. Two FCC prescribed frequency bands have been available for medical telemetry. The VHF (174-216 MHz) spans TV channels $7-13$ and medical telemetry can use frequencies not used by TV. The UHF (450-470 MHz) is below the UHF TV band but medical telemetry is permitted only on a secondary basis to private aland mobile radio services such as high transmitter powered communication $\mathrm{f}$ used by emergency vehicles. A secondary user cannot interfer with the primary emergency mobile units and the secondary user will have to tolerate the interference from those services. The inadequacy of these bands became clear with the introduction of digital television which occupies both VHF and UHF frequencies. In 1999 the FCC introduced two new options: either three bands of $08-614 \mathrm{MHz}, 1395-1400 \mathrm{MHz}$; and 1429-1432MHz ; or two bands 608-614 MHz and 1391-1400 MHz. The FCC will authorize medical telemetry equipment restricted to the allowed bands.

Practical local area networks are not limited by the FCC prescribed frequency bands but are subject to interference from noise (e.g.,cell phones) transmission coverage, multipath data blurring. Below some advantages of multiple frequency systems are discussed using both $2.5 \mathrm{GHz}$ and $4 \mathrm{XX} \mathrm{MHz}$ frequencies.

The three methods of wireless transmission to be considered in transmitting biomonitor and biosensor information are radio frequency electromagnetic signals, infrared optical signals and acoustic signals. The main focus of most wireless transmission is RF, but optical data transmission can be efficient and interference proof in some applications. Acoustic transmission of data over phone lines from EKG data devices has shown some applications (28) but this mode will not be discussed further in this review.

One of the commercial devices appropriate for ambulatory monitoring of ECG and blood oxygenation through pulse oximetry is the Guardian Telemetry Transmitter Model 20601 which transmits in the 450$470 \mathrm{MHz}$ range and costs about $\$ 2500$. Improved models through minituration and alternative methods of acquiring heart rate and pulse wave form using a personal LAN can be expected for these higher transmitter power ambulatory devices. 
Budinger TF. Biomonitoring with wireless communications. Annu Rev Biomed Eng 2003; 5:383-412.

Demonstration of the global capabilities of wireless communication of vital sign information was an experiment from an airborne Boeing 757 to three remote locations on the ground (29). Because all recipient stations relied on an institutional network to receive the information, it was not possible to transfer data to a given location beyond the hospital campus. This limitation can be overcome using Wireless Application Protocol (WAP) technology for the Internet. Cellular Digital Packet Protocols (CDPD) enabled data transfer speeds up to 19,200 bps to a digital cellular phone (G2). Medical data included blood pressure, pulse, respiratory rate, end tidal $\mathrm{CO} 2$, oxygen saturation and EKG tracings were transferred from a G2 (digital cellular) linked to a hand-held computer.

\section{RF transponders}

Most systems be deployed use a simplex reporting radio which requires minimum on-board battery drain. An advance over these systems is a duplex device which can be controlled remotely so that an individual's status can be interrogated by querying the on-board central processor unit for data in order to down-load, set sensitivity, deploy filters or engage alternate biosensors available on or implanted in the patient. An example of a duplex system operating at $2.45 \mathrm{GHz}$ using a hospital LAN depends on a transponder uplink (base station to patient) with $2.45 \mathrm{GHz}$ selected as the interrogating frequency and $418 \mathrm{MHz}$ as the downlink (patient to base) for sending biosignals. The downlink uses well-established SAWR circuitry (30).

\section{Antennas}

Systems requirements for selection of the optimal frequencies for wireless biomonitoring necessitate evaluation of the tradeoffs between ambient noise frequencies, antenna size, efficiency and polarization, effective reliable communications distances, power available for interrupted or continuous monitoring $(5,31)$. Transmission of downlink signals at $418 \mathrm{MHz}$ has advantages because the keying rates up to 20k-bit/s are available which would allow digital waveform signals to be transmitted (RF Monolithics Inc Dallas Tx). Transmission of the interrogating or uplink message offers advantages of low levels of Biomonitoring page 13 
Budinger TF. Biomonitoring with wireless communications. Annu Rev Biomed Eng 2003; 5:383-412. man made noise, wide bandwidth, ease of directional antenna deployment and potentially higher antenna efficiencies compared to lower UHF and VHF bands.

The free space path loss is given by the Friis formula:

$\operatorname{PL}(\mathrm{dB})=20 \log [\lambda / 4 \pi \mathrm{d}]$

With $2.45 \mathrm{MHz}$ and a distance of 10 meters one expects the free space path loss to be $-33 \mathrm{~dB}$ for a transmitted power of $27 \mathrm{~dB}(0.5 \mathrm{~W})$.

For a range of 1 mile $(1.6 \mathrm{~km})$ and cellular phone frequency of about $900 \mathrm{MHz}(33.3 \mathrm{~cm})$ the power into a matched receiver is $-68 \mathrm{db}$.

\section{Communications Beyond the LAN}

Once the data have reached a downlink node the signals can now be moved to multiple noise impervious and non-lossy hard wire links such as the internet, phone lines into care givers stations, rescue worker stations or concerned friends and family. Transmissions from biosensor to modems to telephone systems and thence to FAX hard copy is a mode useful for two dimensional signals such as 12-lead EKG or images. Somewhat more flexible yet less reliable receivers are cell phones, pocket wireless or palm computers. These modes are illustrated in Figure 9

An aspect of wireless telecommunication allowing caregivers visual, voice, and data communication with patients and health care systems is the development of a wearable personal computer (PC). One proposed system has a core technology based upon a wearable personal computer with a smart-card interface coupled with speech, pen, video input and wireless internet connectivity. The TransPAC system with the MedLink software system is designed to provide an integrated solution for a broad range of health care functions where mobile and hands-free or limited-access systems are preferred or necessary and where the capabilities of other mobile devices are insufficient or inappropriate. For 
Budinger TF. Biomonitoring with wireless communications. Annu Rev Biomed Eng 2003; 5:383-412. example, a web browser-like display, accessible through either a flatpanel, standard, or headset monitor, gives the beltpack TransPAC computer the functions of a complete desktop including PCMCIA card interfaces for internet connectivity and a secure smartcard with 16-bit microprocessor and upwards of 64K memory (32).

\section{Personal Image Transmission}

Though not strictly biomonitoring in the theme of this review, we would be negligent not to present prospects for communicating a patient's status through colored images of face, wounds, rashes, or environmental situations requiring attention or advice from caregivers or rescuers. The general concept is shown in Figure 10. The current activities in the area of transmission of medical images has involved experiments to determine the feasibility and weaknesses of transmitting EKG data or X-Ray CT image data through the internet or through a commercial wireless network to a hand-held or pocket sized display unit. High bandwidth cellular phones being introduced to access the internet (e.g. Nokia 7110 Mobile Application Protocol (WAP) phone) as well as currently available pocket computers have the capacity to receive two dimensional images ranging from radiological images to images of people, places or things (e.g. wounds, skin rashes) that can be acquired by miniature digital cameras. The current experience is with only a few commercially available devices: Pocket Computers and Personal Data Assistants described below. 
Budinger TF. Biomonitoring with wireless communications. Annu Rev Biomed Eng 2003; 5:383-412.

Two units currently available are:

4) Hewlett Packard (HP; PaloAlto, CA) 620LX is a pocket computer with a 256 color 640 by 240 pixel screen, measuring 19.5 by 10.0 by $3.2 \mathrm{~cm}$, weighing 603 grams.

5) The Sharp Mobilon 4500 (Sharp, Mahwah, NJ) is a pocket computer with a 256 color 640 by 240 pixel screen, measuring 18.5 by 9.7 by $3.0 \mathrm{~cm}$, weighing 483 grams.

Both pocket computers run under the Microsoft Windows CE operating system and come preloaded with a set of software which includes Microsoft Pocket Internet Explorer, a world wide web (WWW) browser (Microsoft, Redmond, WA)

Current experience with the transmission and reception of X-ray CT scans demonstrated the feasibility in a study with images from 21 patients but also showed the major limitation of 21 minutes for and average of 14 images (40kBytes each) for each case.

Since connection initialization, transfer and reception times are too long because this experiment used only 9,600 baud. We should expect rates of 57.6 baud which in principle would reduce the reception of a single image from $90 \mathrm{~s}$ to $15 \mathrm{~s}$.

Wireless connections between these pocket computers and the internet through a telecommunications company's cellular digital packet data (CDPD) network requires a modem such as the Sierra Wireless Air Card 300 or 555(Sierra Wireless Inc. Richmond. BC, Canada; www.sierrawireless.com) as a CDPD modem. That credit card size device with a $7 \mathrm{~cm}$ antenna which extends to $11 \mathrm{~cm}$ can in principle operate at transmission speeds of 19,200 baud.(model 300) or 152,000 baud (model 555).

Computers smaller than palmtops, known as PDA's (personal digital assistants), have recently developed screens of high enough quality to satisfactorily display a CT scan. PDAs are about two-thirds the size of a palmtop computer making the PDA more portable. Most PDA computers cannot yet accept 
Budinger TF. Biomonitoring with wireless communications. Annu Rev Biomed Eng 2003; 5:383-412. any of the available wireless modem cards on the market. It is very likely that a wireless modem card for a PDA will soon be available or a new color PDA will become available that will accept existing wireless modem cards such as Sierra Wireless AirCard 300. An example of a PDA which can receive and display images is The Nokia 9000(Nokia Ltd, Helsinki, Finland) is a PDA terminal device for image reception and viewing when combined with a GSM digital phone with internet capabilities is a medium size hand-held device $(17.3 \times 6.4 \times 3.8 \mathrm{~cm})$ with a liquid crystal display of eight gray levels and $600 \mathrm{x}$ 200 pixels. Memory of 8 Mbytes with 2 M bytes for user data storage an accommodate JPEG compressed radiologic optical images which can by 40kbytes. The acquired image data are nominally $256 \times 256$ or 512 x 512 (256kByte) and a reasonable JPEG quality factor is $75 \%$.

\section{Glossary}

ARDIS - ARDIS company, Lincolnshire, IL. A network that is exclusively designed for wireless data transmission, yet its maximum speed is still only 19,200 baud using its available wireless card modem.

BSWD - Bell South Wireless Data. A company similar to ARDIS in that it is also exclusively designed for wireless data transmission.

CDMA - code division multiple access. A method of wireless telephone voice communication. It is a digital cellular technology that uses spread-spectrum techniques.

CDPD - cellular digital packet data. A method of wireless data communication transmitted over cellular telephone networks.

CSTN - color super-twist nematic. A type of flat display screen that is generally used on laptop computers to display a passive matrix color screen, which is generally of lower quality compared with an active matrix color screen.

D-AMPS - Digital Advance Mobile Phone Systems, United States of America based 
Budinger TF. Biomonitoring with wireless communications. Annu Rev Biomed Eng 2003; 5:383-412.

DSTN - double-layer super-twist nematic. A type of flat display screen that is generally used on laptop computers to display a passive matrix color screen. It uses two display layers to counteract the color shifting that occurs with conventional super-twist displays.

EDGE - Enhanced data for GSM evolution

ETSI - European Telecommunications Standards Institute

FTP - File transfer protocol.

GPRS - General packet radio service

GPS - Global Positioning System - The existing GPS radio wave bands are L1 at $1575.42 \mathrm{MHz}$ and L2 at 1227.6 MHz.

GSM - Global Systems for Mobile Telecommunications. A digital wireless system which was initially developed for digital voice telephones, but it is also capable of digital data transmission at a modest speed of 9,600 baud. GSM is expected to go far beyond $9.6 \mathrm{~Kb}$.

Iridium - Iridium world communications. Provides worldwide wireless communication coverage using a network of land-based and satellite-based communications. Iridium customers can be reached anywhere on the globe, but coverage is not reliable indoors in many areas.

JPEG - Joint Photographic Expert Group. This is a means of compressing the size of computer images so they require less time to transmit and less storage space.

LAN - Local area network designates communication system which could be on the subject between a sensor and a CPU located on the belt for example with subsequent transmission from the CPU to another LAN or regional communication system such as the cellular network.

PC - Personal computer.

PCMCIA - Personal computer memory card international association. A computer connection standard for card sized computer devices to attach to a portable computer by inserting it into a "PCMICIA" slot.

Biomonitoring

page 18 
Budinger TF. Biomonitoring with wireless communications. Annu Rev Biomed Eng 2003; 5:383-412.

PDA - Personal digital assistant. A very small pocket computer.

PPP - Point-to-point protocol

RAS - Remote access service

RGB - red green blue. The three color dot elements that create all the colors on a computer or television screen.

TDMA - time division multiple access. A technology for delivering digital wireless service using time-division multiplexing. TDMA is used by the GSM digital cellular system.

TCP/IP - Transmission control protocol/internet

TFT - thin film transistor. A type of flat screen display that is generally used on pocket and laptop computers to display an active matrix color screen, which is generally of higher quality compared with a passive matrix color screen.

WAAS - the Wide Area Differential Augmentation System (WAAS or WADGPS) is meant to augment Standard Positioning Services over wide areas. It encompasses a \$500 million network of stations to measure and transmit DGPS corrections via satellite. WAAS-distributed differentials reinforce the availability, accuracy and integrity of signals over a wide area.

WWW - world wide web. A system of internal servers that support specially formatted documents written in html (Hyper Text Markup Language) which permits display of text, graphics, audio, video, and so on, moving from one document or web site to another by clicking on highlighted text (known as Hyper Text) or icons.

\section{Acknowledgements}

This research was supported by the National Institutes of Heart, Lung and Blood (NIH) and the Center for Information Technology Research in the Interest of Society (CITRIS) at the University of California, Berkeley. I thank Dr. Kathleen Brennan for her assistance with the illustrations and the preparation of this manuscript. 


\section{Figure Legends}

Figure 1 Synopsis of portable non-invasive and easily worn monitoring systems with wireless potentials

Figure 2: Signals from the ECG and pulse pressure changes provide different physiologic information and can be used together to gain additional data such as the pulse transit time (PTT). Rapid pulse timing can be from a pulse pressure monitor or from a pulse oximeter in the reflectance mode or conventional transmission mode.

Figure 3. The portable Minolta Pulsox 3 wrist pulse oximeter ( $\$ 580$ US).

Figure 4: Expanded view of a transcutaneous carbon dioxide sensor. Heating the skin promotes arterialization.

Figure 5: Illustration of a self contained, wrist worn blood pressure device similar to that marketed by OMRON Inc ( $\sim 75$ US)

Figure 6. General concept of a wearable accelerometer or motion detector which allows continuous monitoring of a subjects daily activity as well as detection of a fall. Wireless communication of information can be stored in a local network node or the belt worn transmitter can be used to transmit alert signals or as a relay for actual data.

Figure 7: A customized tracking system for private automobile or a wandering family member can be a major household appliance but currently relies on HAM radio licensed operations. 
Budinger TF. Biomonitoring with wireless communications. Annu Rev Biomed Eng 2003; 5:383-412.

Figure 8: Designed for pilots and air crews, this Breitling watch has a built-in micro-transmitter which is activated by unscrewing a protective cap and pulling the antenna out fully. The system will broadcast at 121.5 MHz emergency frequency for 48 hours.

Figure 9 Multimodal communication of medical information for improving availability to caregivers and medical consultants is evolving in step with improvements in technologies. At the left is shown a consultant encumbered with multimodal communications. At the right is shown the current capability of hand-held communication devices for viewing data as well as radiologic images.

Figure 10 Voice, personal image, whereabouts and emergency data communication for the patient at risk can in principle be supplied by a miniature camera, LAN and regional network. 


\section{Literature Cited}

1. Neuman MR, Picconnatto JRoux JF. 1970. A wireless radiotelemetry system for monitoring fetal heart rate a intrauterine pressure during labor and delivery. Gynecol Invest. 1: 92-104

2. 2000. Physiologic monitoring systems. Health Devices. 29: 153-84.

3. 1998. Medical instrumentation : application and design. New York: John Wiley and Sons Inc.,

4. 2000. Introduction to Bioengineering. Oxford, New York: Oxford University Press,

5. Riemann AIEvans NE. 1999. The incidence of radio-frequency impulsive noise within hospital buildings: initial measurements in the $450 \mathrm{MHz}$ band. Medical Engineering \& Physics. 21: 569-74

6. Ti PM, Yamamoto LGShirai LK. 2001. Instant telemedicine ECG consultation with cardiologists using pocket wireless computers. Am J Emerg Med. 19: 248-9.

7. Johnson P, Andrews DC, Wells S, de Lusignan S, Robinson JVandenburg MJ. 2001. The use of a new continuous wireless cardiorespiratory telemonitoring system by elderly patients at home. Telemed Telecare. 7: 76-7

8. Satava R, Angood PB, Harnett B, Macedonia CMerrell R. 2000. The physiologic cipher at altitude: telemedicine and real-time monitoring of climbers on Mount Everest. Telemed J E Health. 6: 30313.

9. Gandsas A, Montgomery K, McKenas D, Altrudi RSilva Y. 2000. In-flight continuous vital signs telemetry via the Internet. Aviat Space Environ Med. 71: 68-71.

10. Harnett BM, Doarn CR, Russell KM, Kapoor V, Merriam NRMerrell RC. 2001. Wireless telemetry and Internet technologies for medical management: a Martian analogy. Aviat Space Environ Med. 72: 1125-31. 
Budinger TF. Biomonitoring with wireless communications. Annu Rev Biomed Eng 2003; 5:383-412.

11. Severinghaus JWNaifeh KH. 1987. Accuracy of response of six pulse oximeters to profound hypoxia. Anesthesiology. 67: 551-8.

12. Nogawa M, Kaiwa TTakatani S. 1998. A novel hybrid reflectance pulse oximeter sensor with improved linearity and general applicability to various portions of the body. Proceedings of 20th Annual International Conference of the IEEE Engineering in Medicine and Biology Society. Vol.20 Biomedical Engineering Towards the Year 2000 and Beyond (Cat. No.98CH36286). HK Chang and YT Zhang, eds. Hong Kong, China, 20:1858-61

13. Huch A, Lubbers DWHuch R. 1973. [Patient monitoring by transcutaneous PCO2-measurement and simultaneous control of the relative local perfusion (author's transl)]. Anaesthesist. 22: 379-80.

14. Herrell N, Martin RJ, Pultusker M, Lough MFanaroff A. 1980. Optimal temperature for the measurement of transcutaneous carbon dioxide tension in the neonate. J Pediatr. 97: 114-7.

15. Tremper KK, Mentelos RAShoemaker WC. 1981. Clinical and experimental transcutaneous pCO2 monitoring. j Clin Eng. 6: 143-147

16. Pitson D, Chhina N, Knijn S, van Herwaaden MStradling J. 1994. Changes in pulse transit time and pulse rate as markers of arousal from sleep in normal subjects. Clin Sci (Lond). 87: 269-73, Davies RJ, Crosby J, Vardi-Visy K, Clarke MStradling JR. 1994. Non-invasive beat to beat arterial blood pressure during non-REM sleep in obstructive sleep apnoea and snoring. Thorax. 49: 335-9, Smith RP, Argod J, Pepin J-LP.A. L. 1999. Pulse Ttansit Time: an appraisal of potential clinical applications. Thorax. 54: 452-7

17. Urschitz MS, Eichler F, Popow CIpsiroglu OS. 2000. [Evaluation of the cardiorespiratory monitor SpiroGuard $\mathrm{C}$ for infants. Improved registration of respiratory events by new sensors and intelligent alarm management system]. Wien Klin Wochenschr. 112: 226-33. 
Budinger TF. Biomonitoring with wireless communications. Annu Rev Biomed Eng 2003; 5:383-412.

18. Neuman MRO'Connor E. 1980. A two-channel radiotelemetry system for clinical fetal monitoring. Biotelem Patient Monit. 7: 104-21, Neuman MR, Watson H, Mendenhall RS, Zoldak JT, Di Fiore JM, Peucker M, et al. 2001. Cardiopulmonary monitoring at home: the CHIME monitor. Physiol Meas. 22: 267-86.

19. Gurley RJ, Lum N, Sande M, Lo BKatz MH. 1996. Persons found in their homes helpless or dead. N Engl J Med. 334: 1710-6.

20. Wilson LS, Gill RW, Sharp IF, Joseph J, Heitmann SA, Chen CF, et al. 2000. Building the Hospital Without Walls--a CSIRO home telecare initiative. Telemed J. 6: 275-81.

21. Williams G, Doughty KBradley DA. 2000. Safety and risk issues in using telecare. J Telemed Telecare. 6: 249-62

22. Hall EHK. 1991. Biosensors. Englewood Cliffs, N.J.: Prentice Hall, , 1997. Handbook of biosensors and electronic noses : medicine, food, and the environment. Boca Raton: CRC Press,

23. Hosli P, Avrameas S, Ullmann A, Vogt ERodrigot M. 1978. Quantitative ultramicro-scale immunoenzymic method for measuring Ig antigenic determinants in single cells. Clin Chem. 24: 1325-30.

24. Geiss A, Varadi E, Steinbach K, Bauer HWAnton F. 1997. Psychoneuroimmunological correlates of persisting sciatic pain in patients who underwent discectomy. Neurosci Lett. 237: 65-8.

25. Streckfus CF, Johnson RB, Nick T, Tsao ATucci M. 1997. Comparison of alveolar bone loss, alveolar bone density and second metacarpal bone density, salivary and gingival crevicular fluidinterleukin-6 concentrations in healthy premenopausal and postmenopausal women on estrogen therapy. J Gerontol A Biol Sci Med Sci. 52: M343-5 
Budinger TF. Biomonitoring with wireless communications. Annu Rev Biomed Eng 2003; 5:383-412.

26. Gilchrist W, Burkhalter E, Eaton C, Schaudies RP, Maydonovitch C, Andrada F, et al. 1994. The effect of indomethacin on the secretion of human salivary epidermal growth factor. Am J Gastroenterol. 89: 97-100.

27. Perez Navero JL, Jaraba Caballero S, Ibarra de la Rosa I, Jaraba Caballero MP, Guillen del Castillo M, Montilla Lopez P, et al. 1999. Effects of competitive physical exercise on neuroendocrine response interleukin-6 liberation in children. An Esp Pediatr. 51: 267-72

28. Orlov OI, Drozdov DV, Doarn CRMerrell RC. 2001. Wireless ECG monitoring by telephone. Telemed J E Health. 7: 33-8.

29. Gandsas A, Montgomery K, McIntire KAltrudi R. 2001. Wireless vital sign telemetry to hand held computers. Stud Health Technol Inform. 81: 153-7

30. Scanlon WG, Evans NE, Crumley GCMcCreesh ZM. 1996. Low-power radio telemetry: the potential for remote patient monitoring. J Telemed Telecare. 2: 185-91

31. Kajiwara A. 1994. On a circular polarization wave transmission in LOS indoor radio channels. Proceedings of Wireless Networks - Catching the Mobile Future - 5th IEEE International Symposium on Personal, Indoor and Mobile Radio Communications. JH Weber, JC Arnbak and R Prasad, eds. Amsterdam, Netherlands, 1:156-9, Hashemi H. 1993. The indoor radio propagation channel. Proceedings of the IEEE. 81: 943-68

32. Koval TDudziak M. 1999. MediLink: a wearable telemedicine system for emergency and mobile applications. Stud Health Technol Inform. 64: 93-107 


\section{Figures}

\section{Biomonitoring}

\section{GPS}

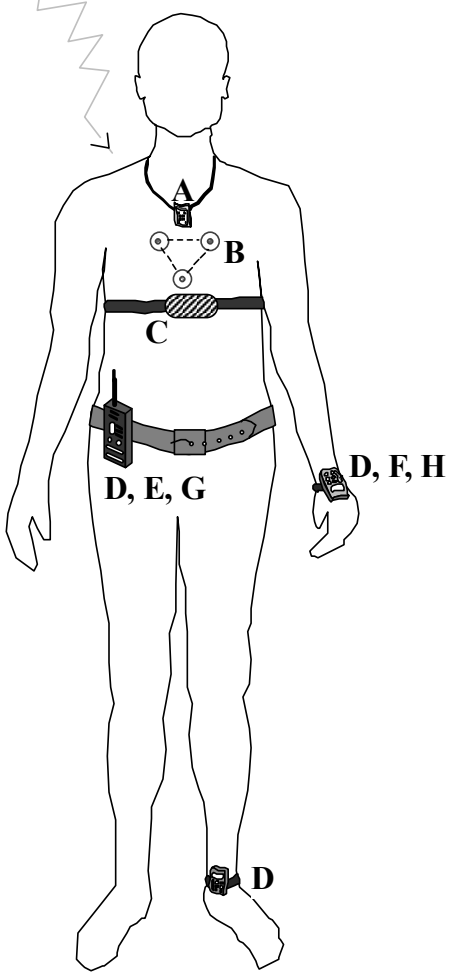

A. Chemistry chip pendant (saliva sampling)

B. Skin electrodes

(cardiac \& respiratory)

C. Strain guage \& Heart Rate Monitor

D. Accelerometer

E. Phone / Camera / GPS

F. Chemistry

$\left(\mathrm{p0}_{2}, \mathrm{pCO}_{2}\right.$, sugar $)$

G. Local area network

H. Pulse Pressure

Figure 1

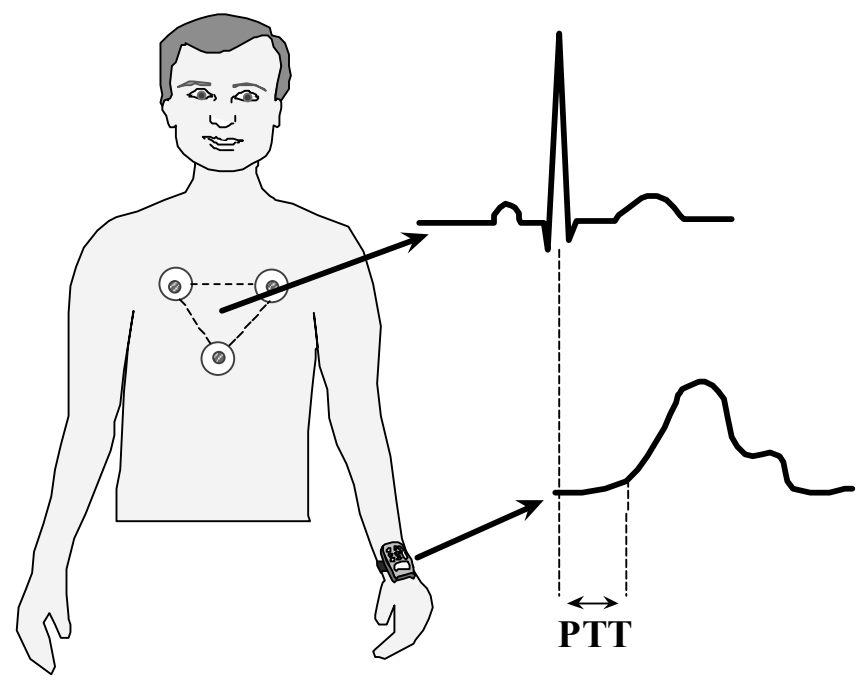

Figure 2: 
Figure 3.
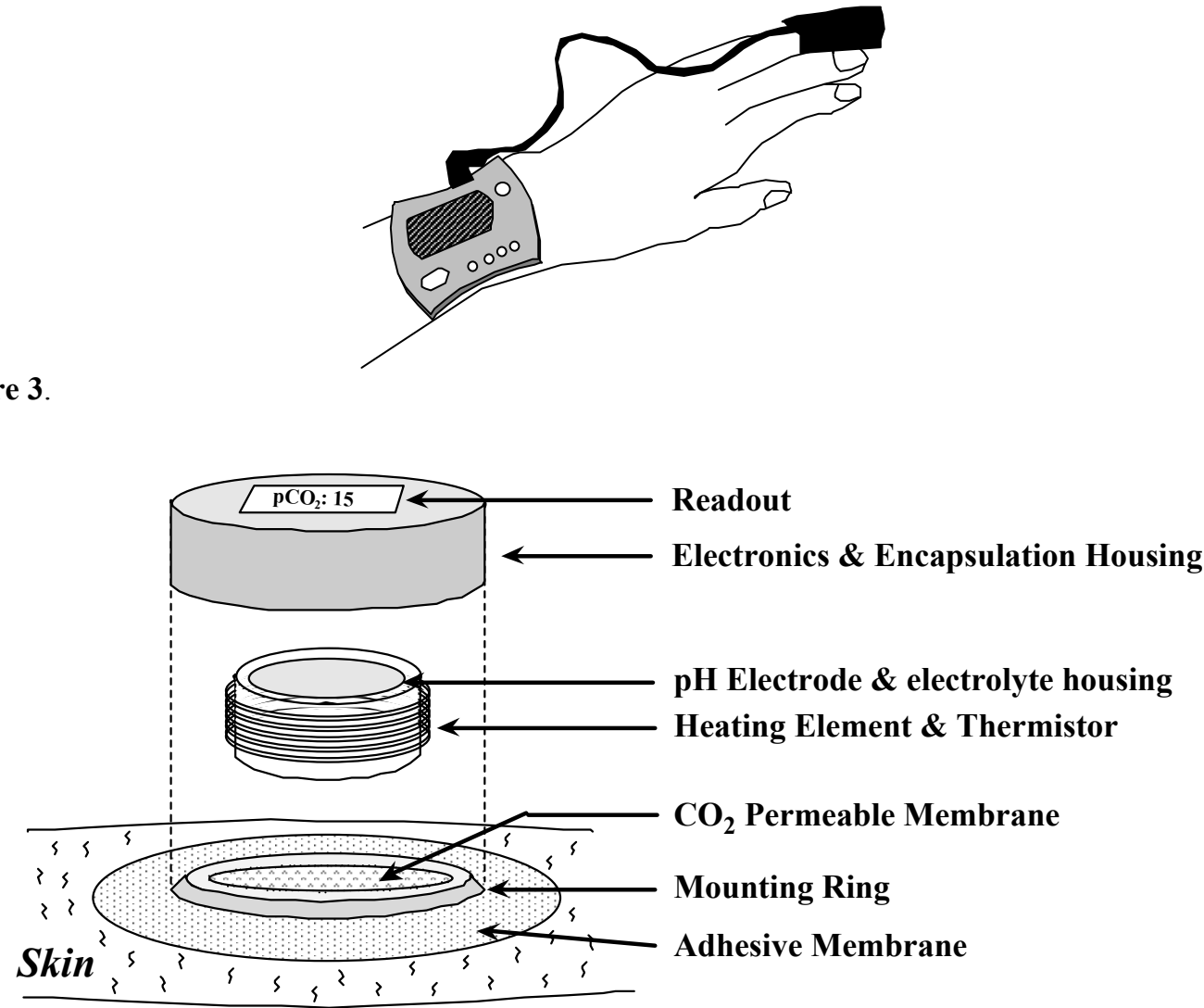

Figure 4:

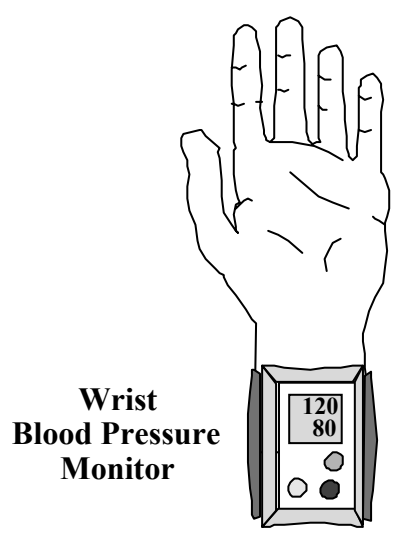

Figure 5: 
Budinger TF. Biomonitoring with wireless communications. Annu Rev Biomed Eng 2003; 5:383-412.

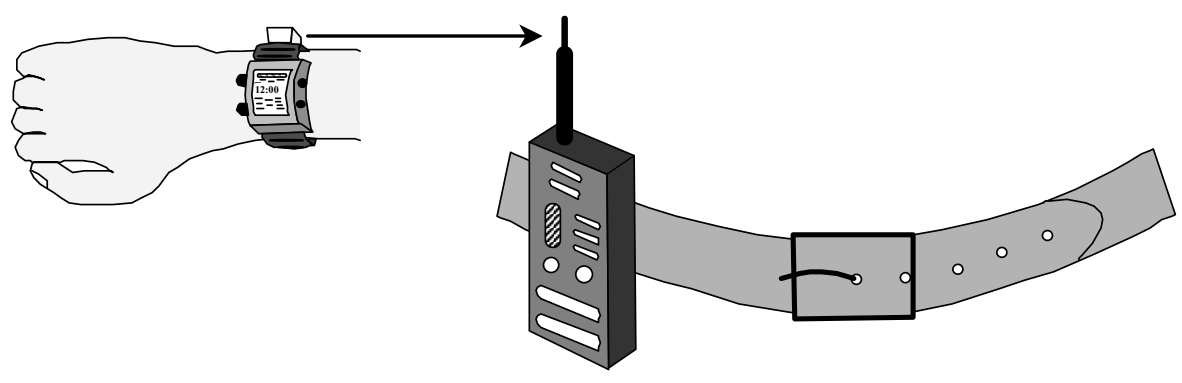

Figure 6.

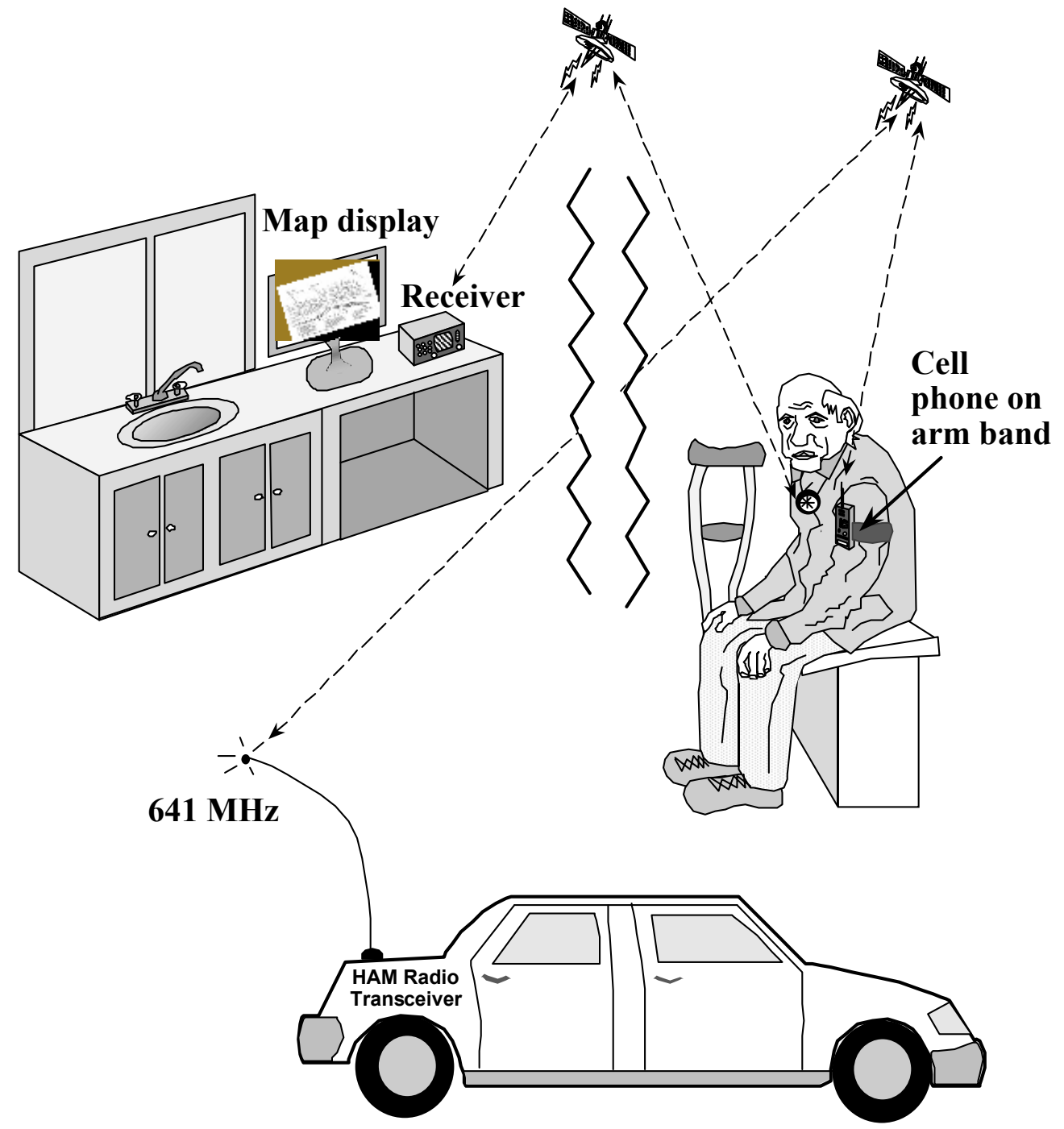

Figure 7: 
Budinger TF. Biomonitoring with wireless communications. Annu Rev Biomed Eng 2003; 5:383-412.

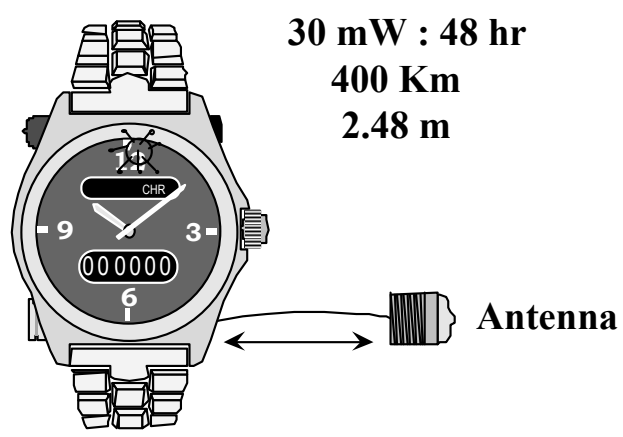

Figure 8: 
Budinger TF. Biomonitoring with wireless communications. Annu Rev Biomed Eng 2003; 5:383-412.
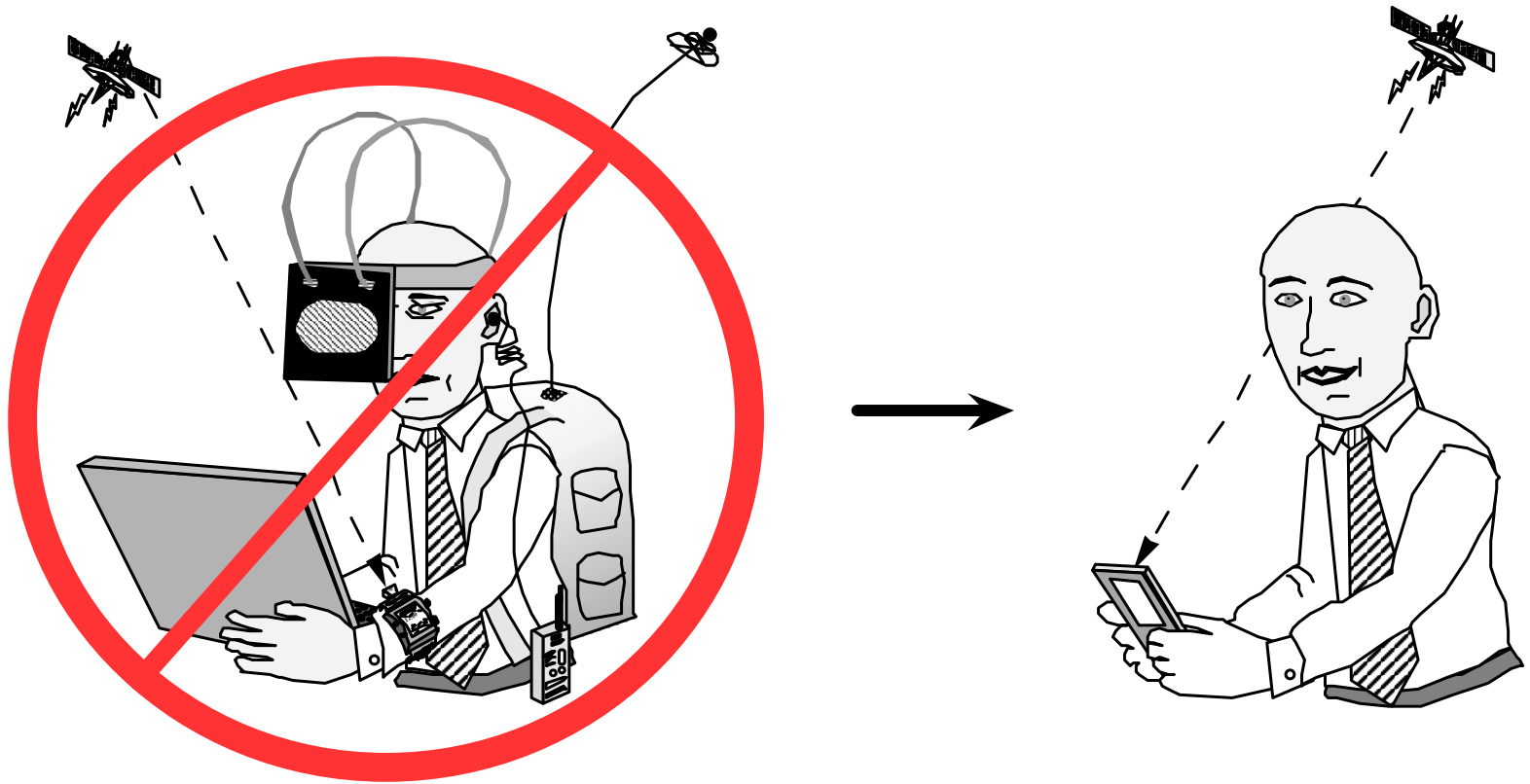

Figure 9

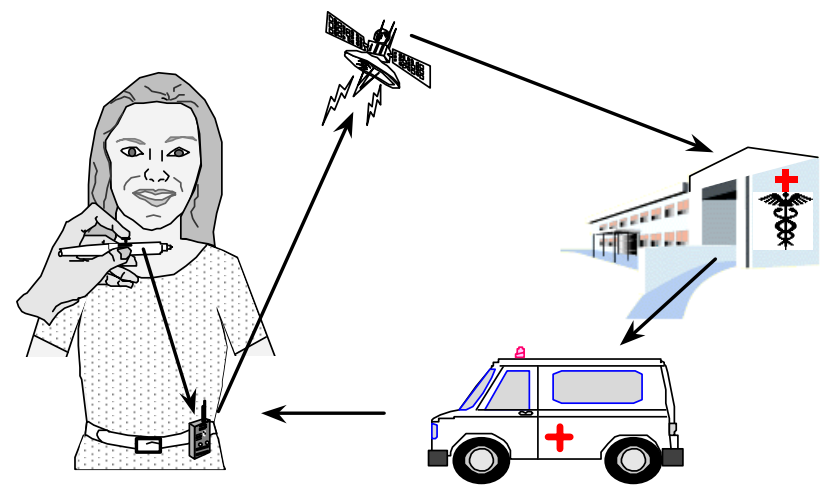

Figure 10 